\title{
BIMA Sub-arcsecond Carbon Monoxide Observations: Resolving the Inner kpc Region of the QSO I Zw 1
}

\author{
Johannes Staguhn ${ }^{1}$ \\ Dept. of Astronomy, University of Maryland, College Park, MD 20742, \\ $U S A$
}

\author{
Eva Schinnerer \\ Dept. of Astronomy 104-24, California Institute of Technology, \\ Pasadena, CA 91125, USA \\ Andreas Eckart \\ I. Physikalisches Institut, Universität zu Köln, Zülpicher Straße 77,
50937 Köln, Germany
}

\begin{abstract}
We present the first sub-kpc $\left(\sim 0.7^{\prime \prime} \approx 0.8 \mathrm{kpc}\right)$ resolution ${ }^{12} \mathrm{CO}(1-0)$ observations of the ISM in the host galaxy of the QSO I Zw 1 which were obtained with the BIMA mm-interferometer in its A configuration. The measurements, which are part of a multi-wavelength study of I Zw 1, will allow comparison of the ISM properties of a QSO host with those of nearby galaxies and place constraints on galaxy formation/evolution models. Our maps of the ${ }^{12} \mathrm{CO}(1-0)$ line emission from the host galaxy of a QSO show a ring-like structure in the circumnuclear molecular gas distribution with a radius of about $900 \mathrm{pc}$. The presence of such a molecular gas ring was already predicted from earlier lower angular resolution PdBI observations (Schinnerer, Eckart, \& Tacconi 1998). A first comparison of the BIMA data with new $\mathrm{PdBI}{ }^{12} \mathrm{CO}(2-1)$ observations with $0.9^{\prime \prime}$ angular resolution shows variations in the excitation conditions of the molecular gas in the innermost 3" comprising the nuclear region of I Zw 1.
\end{abstract}

\section{Introduction}

High resolution molecular line observations of galactic nuclei allow a detailed study of the kinematics of the cold interstellar medium and the derivation of important parameters such as gas masses, surface mass densities and star formation efficiencies. Observations with sub-kpc resolution are required for the separation of starburst and AGN components in the nuclei of active galaxies which is a key problem in the investigation of evolutionary sequences proposed for AGNs (Norman \& Scoville 1988; Sanders et al. 1988; Rieke, Lebofsky, \&

\footnotetext{
${ }^{1}$ Current address: Code 685, NASA - Goddard Space Flight Center, Greenbelt, MD 20771, USA
} 
Walker 1988). Consequently there is a large sample of high resolution observations available for nearby objects. These observations have revealed the presence of circum-nuclear starburst rings in a large number of (nearby) active and IR luminous galaxies (e.g. NGC 1068: Planesas, Scoville, \& Myers 1991; NGC 7469: Genzel et al. 1995). The extension of high angular resolution observations of molecular gas emission lines to the host galaxies of QSO is essential to understand the connection between the local active galaxies and high-z QSOs.

Due to the limited angular resolution of single dish $\mathrm{mm}$ wavelength telescopes, only interferometric observations at this wavelength allow insight into the morphology and into the kinematics of molecular clouds in the nuclear region of QSO host galaxies. In the following we present $\sim 0.7$ " angular resolution ${ }^{12} \mathrm{CO}(1-0)$ observations of the ISM in the host galaxy of the QSO I Zw 1 . The host galaxy of this QSO was observed before with the PdBI at lower angular resolution in ${ }^{12} \mathrm{CO}(1-0)$ (Schinnerer et al., 1998) and in ${ }^{12} \mathrm{CO}(2-1)$ (Eckart, Schinnerer, \& Staguhn, these proceedings). Position-velocity diagrams of the $\mathrm{PdBI}{ }^{12} \mathrm{CO}(1-0)$ observations along the major axis of $\mathrm{I} \mathrm{Zw} 1$ indicated the presence of a ring-like molecular gas feature around the nucleus with a radius of about 0.8 ". However, the angular resolution of these observations is insufficient to resolve the feature in the individual channels. The PdBI ${ }^{12} \mathrm{CO}(2-1)$ observations, on the other hand, show the molecular line emission to be centrally peaked, but the 0.8 " molecular ring is not evident in the data. Here we present the first mm-wavelength observations with sub-kpc resolution of the host galaxy of a QSO.

\section{Observations}

The observations were made in December 1999 in the compact A array configuration (A-) of the ten element BIMA ${ }^{2}$ array (Welch et al. 1996). I Zw 1 has a redshift of $\mathrm{z}=0.0611$ (Condon, Hutchings, \& Gower, 1985). The redshifted ${ }^{12} \mathrm{CO}(1-0)$ frequency is $108.633 \mathrm{GHz}$. The sky has a significantly lower opacity at this frequency than at the rest frequency of ${ }^{12} \mathrm{CO}(1-0)$. The angular resolution we achieve in this configuration is roughly 0.4 " which corresponds a spatial resolution of about $0.5 \mathrm{kpc}$. We employed fast phase referencing: the observations were switched between the source, the phase calibrator and an additional quasar which serves as a reference to determine the accuracy of the determined phases. The duration of one such cycle was about two minutes in order to closely monitor the atmospheric phase variations (Holdaway \& Owens 1993).

\section{Discussion}

Schinnerer et al. (1998) find that the $N_{\mathrm{H}_{2}} / I_{C O}$ conversion factor in I $\mathrm{Zw} 1$ is close to $210^{20} \mathrm{~cm}^{-2} \mathrm{~K}^{-1} \mathrm{~km}^{-1} \mathrm{~s}$ which is also found for molecular gas in our galaxy and in many nearby external galaxies. They observe a ${ }^{12} \mathrm{CO}(1-0)$ line flux density of $56 \mathrm{mJy}$ for the innermost 3.3 " $(3.7 \mathrm{kpc})$ which yields a molecular hydrogen column density of $4.2 \times 10^{22} \mathrm{~cm}^{-2}$ in the nuclear region of I Zw 1 .

\footnotetext{
${ }^{2}$ The BIMA observatory is supported by the NSF grant AST-9981289
} 
The corresponding molecular gas mass is $7.5 \times 10^{9} \mathrm{M} \odot$. The BIMA ${ }^{12} \mathrm{CO}(1-0)$ observations, smoothed to an angular resolution of 2 " - which corresponds to the resolution of the PdBI ${ }^{12} \mathrm{CO}(1-0)$ observations - show a peak flux of $55 \mathrm{mJy}$, consistent with the PdBI observations. There is however an offset of roughly 1" between the peak positions in the PdBI and BIMA observations which will need to be addressed in a more detailed analysis of the data. The measured flux shows that about $2 / 3$ of the molecular gas mass of $I \mathrm{Zw} 1$ is confined within a radius of $1.1 \mathrm{kpc}$ of the nucleus. Figure 1 (left) shows a composite image of a smoothed HST I-band image of I Zw 1 with superimposed contour lines from the ${ }^{12} \mathrm{CO}(1-0)$ PdBI observations from Schinnerer et al. (1998). Figure 1 (right) shows the BIMA ${ }^{12} \mathrm{CO}(1-0)$ observations smoothed to a resolution of $\sim 0.7$ " in order to decrease the rms in the map. A ring-like structure with radius of roughly 0.8 " is visible in the map of the molecular line emission, confirming its predicted presence (Schinnerer et al., 1998). The peak flux density in this BIMA image is $21 \mathrm{mJy}$. Using the galactic $N_{\mathrm{H}_{2}} / I_{\mathrm{CO}}$ conversion factor (see above) this flux density yields a $N_{H_{2}}$ column density of $6 \times 10^{23} \mathrm{~cm}^{-2}$ which is the average hydrogen column density over the area covered by the synthesized beam - roughly $0.55 \mathrm{kpc}^{2}$. As a result the molecular hydrogen column density in the circum-nuclear sub- kpc region of I $\mathrm{Zw} 1$ is two orders of magnitude larger than the average molecular gas column density of $2 \times 10^{21} \mathrm{~cm}^{-2}$ in the disk region of I Zw 1 (see Schinnerer et al., 1998). The giant dense molecular clouds we observe in the nuclear region are very likely the sites of a massive starburst in the central kpc region of the host (Eckart et al. 1994). A detailed analysis of the kinematics of these molecular clouds, which contribute significantly to the total nuclear mass, will be presented in an upcoming paper (Staguhn, Schinnerer, Eckart, in prep.). Detailed knowledge of the nuclear dynamics will be relevant to the theories of black hole formation (e.g. Norman \& Scoville 1988).

The BIMA observations support the conclusion in Eckart et al. (these proceedings), that the lack of enhanced emission from the $0.8^{\prime \prime}$ molecular ring like structure in the ${ }^{12} \mathrm{CO}(2-1)$ position velocity diagrams has to be attributed to the different excitation conditions for the molecular gas in the very nucleus and in the circum-nuclear region.

\section{References}

Condon, J.J., Hutchings, J.B., \& Gower, A.C. 1985, AJ, 90, 1642

Eckart, A., van der Werf, P.P., Hofmann, R., \& Harris, A.I. 1994, ApJ, 424,627

Genzel et al. 1995, ApJ, 444, 129

Holdaway, M.A., \& Owens, F.N. 1993, ALMA Memo Series, 126).

Neugebauer, G., \& Scoville, N., 1988, ApJ, 325, 74

Norman, C. \& Scoville, N. 1988, ApJ, 332,124

Planesas, Scoville, \& Myers 1991 ApJ, 369, 364

Sanders, D.B., Soifer, , B.T., Elias, J.H., Madore, B.F., Matthews, K.,

Rieke, G.H., Lebowsky, M.J., \& Walker, C.E. 1988, ApJ, 325, 679

Schinnerer, E., Eckart, A., \& Tacconi, L.J., 1998, ApJ, 500, 147

Welch, W.J., et al. 1996, PASP, 108, 93 


\section{Zw 1}

\section{$\mathrm{HST} / \mathrm{PdBI} \quad{ }^{12} \mathrm{CO}(1-0) \quad$ BIMA}

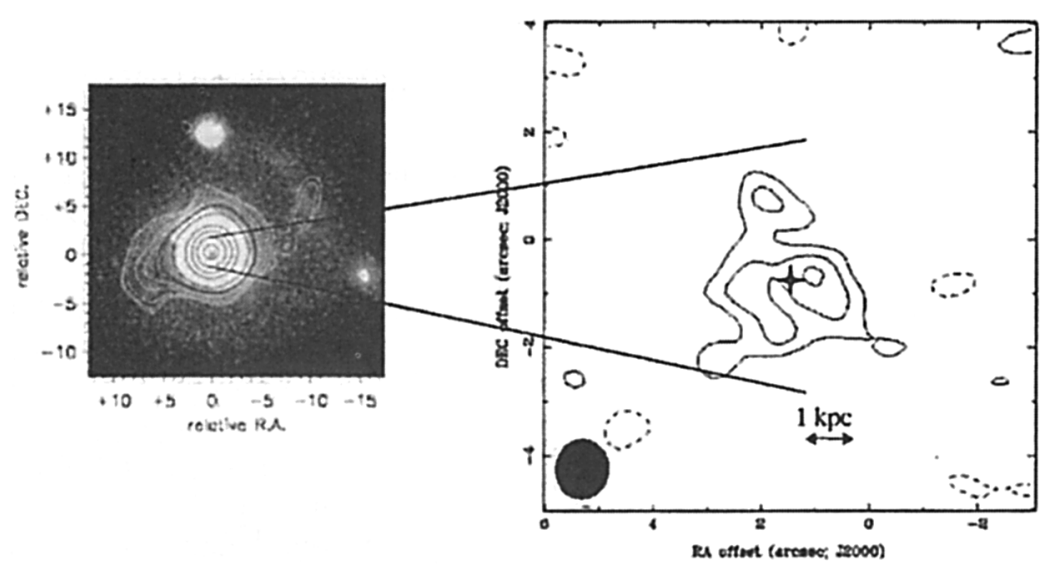

Figure 1. left: contours: PdBI image of the integrated ${ }^{12} \mathrm{CO}(1-0)$ line emission from I Zw 1 with an angular resolution of 5". Underlying the contours is an I band HST image of I Zw 1 convolved to a resolution of 2" for comparison (from Schinnerer et al., 1998). right: BIMA image of the ${ }^{12} \mathrm{CO}(1-0)$ emission smoothed to a resolution of $\sim 0.7 "$. The solid contours are $10 \mathrm{mJy}(2 \sigma), 15 \mathrm{mJy}(3 \sigma)$, and $20 \mathrm{mJy}(4 \sigma)$ over a $400 \mathrm{~km} \mathrm{~s}^{-1}$ velocity interval. The dashed contours represent the corresponding negative values for the flux density. The star indicates the center emission we observe in our ${ }^{12} \mathrm{CO}(1-0)$ BIMA image which is smoothed to 2" resolution, whereas the coordinates indicate the offsets from the PdBI center position. The origin for the offset of about 1 " between the PdBI and BIMA observations is yet unclear. The length of the arrow in the image corresponds to $1 \mathrm{kpc}$ at the distance of $\mathrm{I} \mathrm{Zw} 1$. 